\title{
Medication Reminder and HealthCARE - AN ANDROID APPLICATION
}

\author{
Deepti Ameta, Kalpana Mudaliar and Palak Patel \\ Gandhinagar Institute of Technology, Gandhinagar, Gujarat, India
}

\begin{abstract}
This is an Android-based application in which an automatic alarm ringing system is implemented. It focuses on doctor and patient interaction. Patients need not remember their medicine dosage timings as they can set an alarm on their dosage timings. The alarm can be set for multiple medicines and timings including date, time and medicine description. A notification will be sent to them through email or message inside the system preferably chosen by the patients. They can search doctor disease wise. The patients will get the contact details of doctors as per their availability. Also the users can see different articles related to medical fields and health care tips. The system focuses on easy navigation and good user interface. Many such Medical Reminder Systems have been developed where a new hardware is required but in our work we have made an attempt to develop a system which is economical, time-saving and supports medication adherence.
\end{abstract}

\section{KEYWORDS}

Automatic Alarm, Reminder System, Notification System, Medication Adherence, Medicine Scheduler

\section{INTRODUCTION}

The category of patients involve all human beings-teachers, students, businessmen, housewives, children and also all of us have a busy hectic schedule. Today's life is full of responsibilities and stress. So people are prone to diseases of different types and it is our duty to make ourselves stay fit and healthy. If the patient stays at home then he or she might get someone to look after him/her but when one is not at home, is out of the city or state away from home then it is hard for the family members to call them and remind them their dosage timings every time.

In our developing and technology dependent life we totally rely on gadgets especially smart phones. Today everyone has a smart phone. With this we get an opportunity to use technology in a better way so that it can be made useful to us. And it plays an important part in our daily life and helps us staying fit in many ways.

The remarkable problem is that patients forget to take the proper medicines in proper proportion and in proper time. Medication adherence, which refers to the degree or extent to which a patient takes the right medication at the right time according to a doctor's prescription, has recently emerged as a serious issue because many studies have reported that non-adherence may critically affect the patient, thereby raising medical costs[1]. Medication nonadherence is a common, complex, and costly problem that contributes to poor treatment outcomes and consumes health care resources [2].

DOI : 10.5121/ijmpict.2015.6204 
So we are introducing an Android application whose objective is to remind the patients of their dosage timings through Alarm Ringing system so that they can stay fit and healthy. Through navigation they can search doctors and hospitals and contact details so that they can easily get proper treatment on time. This application focusses on the people who forget to take medicines on time. It allows users to set an alarm along with the fields of date, time and medicine description which will allow them to set alarm for multiple medicines at different time intervals. The notification system will send a notification after setting an alarm. The user can activate or deactivate the notification accordingly. It will be sent as email or message as selected by the user. The patients can search doctor disease wise and area wise which will provide easy searching facility along with doctor's contact information, visiting place and availability time. Medication reminders help in decreasing medication dispensing errors and wrong dosages.

The application is designed on Eclipse. It can be helpful in defence sector and emergency conditions (accidents) and can spread health care awareness. It is life-saving, money saving and time saving application which is easy to use and provides a good user interface.

\section{RELATED WORK}

Many Medication Systems have been developed based upon different platforms and concepts. Use of healthcare related apps is growing but there are many issues related to their functionality. My MediHealth [3] is a medication reminder system for children. It runs on mobile devices such as smart phones, providing user interfaces for configuring medication schedules and user alerts for reminding users about the time and type of medication according to the configured medication schedule. Some systems use sensors, radio-frequency identification (RFID), or motion detection technologies to ensure that patients actually take their medications [4][5][6]. Park et al proposed medication reminder synchronization system based on data synchronization. It transmits OMA (open mobile alliance) DS (data synchronization) based messages containing the patient's medication data and the device configuration data to a remote manager/medical staff. It also synchronizes data (including medication schedules) modified/generated by these personnel in the medication server [1].

Prasad B has discussed the approach of Medicine reminder pro. It is a free application which supports up to 15 reminders. User can select them in either repeating or non-repeating alarm patterns. Any hourly time interval between alarms can be selected, starting from the minimum of 1 hour. At the scheduled time, application will produce a notification with an alarm, vibration or LED indication. [7]

Zao et al have developed Wedjat - Smart Phone Application which tries to avoid medicine administration errors [8].

There are many loopholes of existing reminder systems. To list a few:

They do not provide disease wise searching of the Doctors, no optional notification only compulsion, no facility for scheduling of appointments to the doctors. Some of the systems have a default alarm tone so the users cannot change them. The scheduled reminder suggests any kind of medicine, dose of medicine, etc. automatically without doctor's prescription, which can cause 
harm to the patients. Lastly, many of the systems available require special hardware which need to be purchased.

\section{Proposed SySTEM AND IMPLEMENTATION}

The proposed system is based on Android Operating system which will remind the users to take medicines on time through notification and automatic alarm ringing system.

Android is a Linux-based operating system designed primarily for touch screen mobile devices such as smart phones and tablet computers, developed by Google in conjunction with the Open Handset Alliance. Android was built from the ground-up to enable developers to create compelling mobile applications that take full advantage of all a handset has to offer. The system is specified on android operating system only because the market share of Android is high. [9] Android also comes with an application development framework (ADF), which provides an API for application development and includes services for building GUI applications, data access, and other component types. The framework is designed to simplify the reuse and integration of components. Android apps are built using a mandatory XML manifest file. The manifest file values are bound to the application at compile time. This file provides essential information to an Android platform for managing the life cycle of an application. Examples of the kinds of information included in a manifest file are descriptions of the app's components among other architectural and configuration properties. Components can be one of the following types: Activities, Services, Broadcast Receivers, and Content Providers [10].

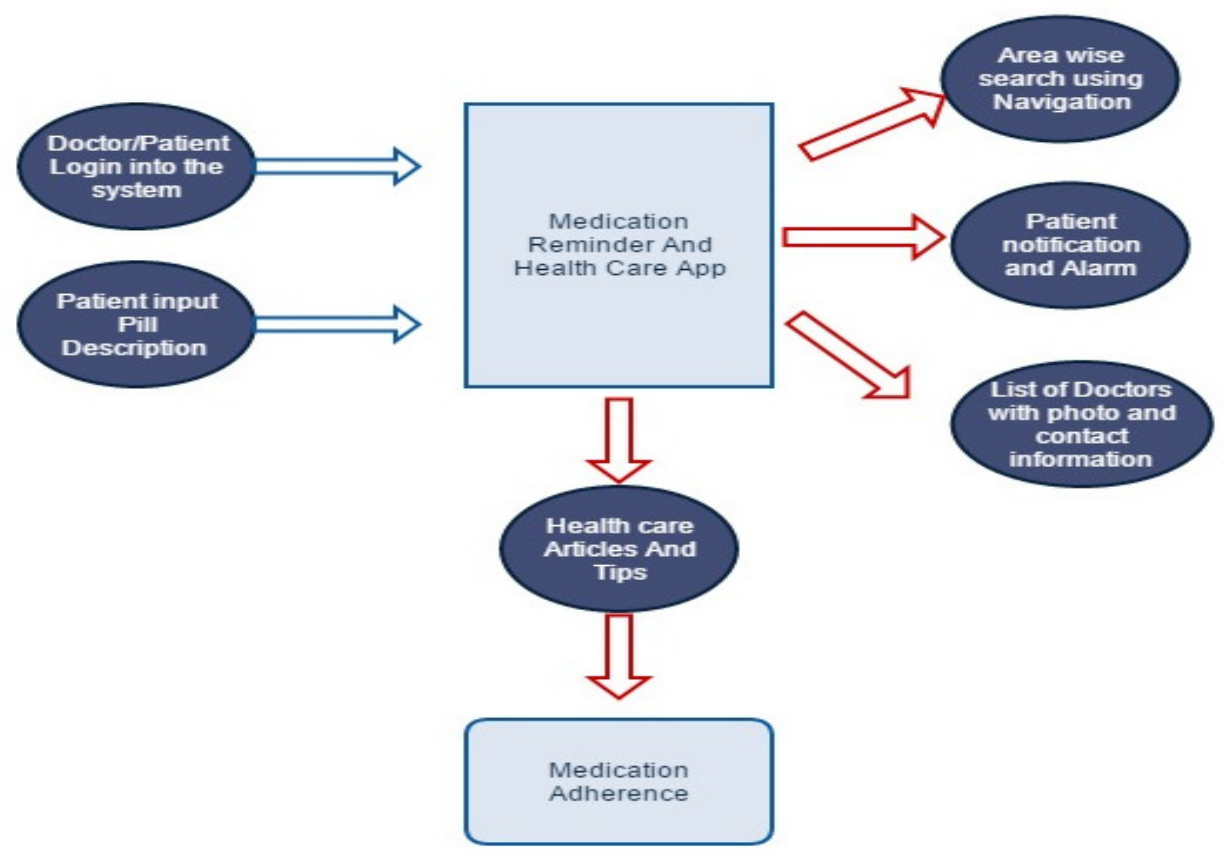

Figure 1. Medication Reminder and Healthcare: System Overview

Figure 1 reflects the overview of the app. Input to the system is the information entered by the patient which includes date, time, medicine name, doctor's name, etc. The output of the system focuses on "Medication Adherence". Medication adherence usually refers to whether patients 
take their medications as prescribed (eg, twice daily), as well as whether they continue to take a prescribed medication. Medication nonadherence is a growing concern to clinicians, healthcare systems, and other stakeholders (eg, payers) because of mounting evidence that it is prevalent and associated with adverse outcomes and higher costs of care [11].

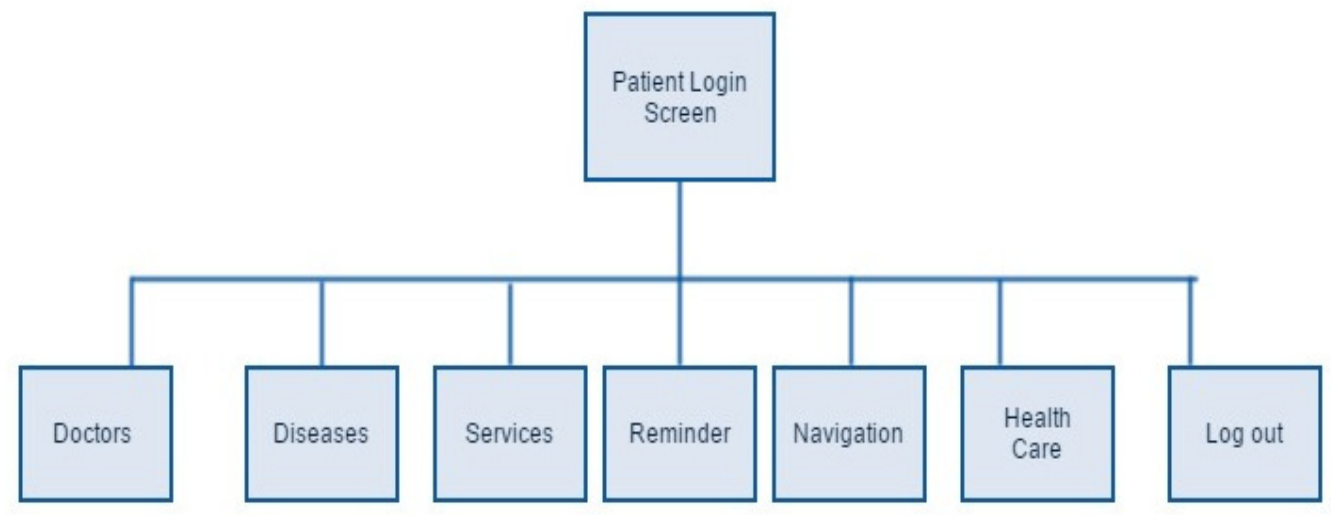

Figure 2. Patient login module

Figure 2 highlights the patient login module. After login the patient will be able to view the list of all the registered doctors with their names, contact information, phone numbers , hospital/clinic address, the availability of doctor accordingly and all other information which the Doctor registers at the time of Signing into the system. They can see the dropdown view of the diseases and can directly navigate to the list of Doctors. It also shows the next appointment with the Doctor. This helps the patients to find the Doctors disease wise. The services help them to understand the system properly so that it becomes useful and productive.

Medication reminders help in decreasing medication dispensing errors and wrong dosages. The Reminder system consists of two parts - setting Alarm and getting notification.

Set Alarm module- It helps in reminding about the medicines. User can add details of his dosage schedules. Using the date field one can enter the starting and ending dates between which he has to take medicines. The time field shows the time of dosage and on that time the alarm will get rung. The user can add the description of the medicine, including name, purpose and other related description. All the information will be saved in the database. This makes any time availability of the patients' records. They can change the ringtone of the alarm from the ringtones stored in the devices. Figure 3 shows this module. 
International Journal of Managing Public Sector Information and Communication Technologies (IJMPICT)

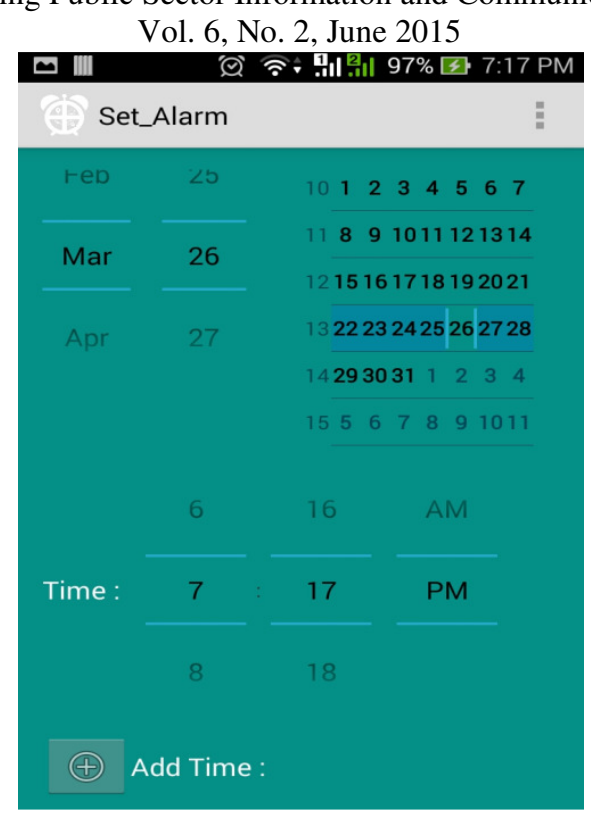

Figure 3. Screenshot of Set_Alarm Module

Get_Notification module: Once the alarm is set then the user gets the notification. The users can activate or deactivate this accordingly. If he does not require the notification he can turn off it. If he requires this system then a notification will be sent into his device. Again if he wants the notification in email form, he can select the 'Notification through Email Mode' or if he requires it in a message format he can go with 'Notification through Message Mode'. Figure 4 depicts the module with Notification through Message Mode.

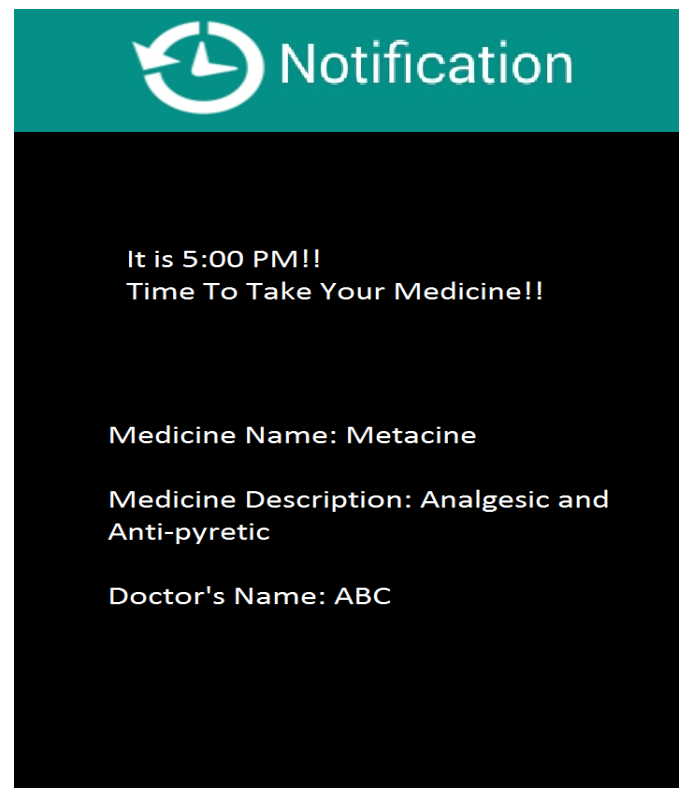

Figure 4. Screenshot of Get_Notification Module (Notification through message mode)

Healthcare Module: In it, the patients can read different posts, articles, new technology in medical sciences, tips and other information of staying fit because staying fit is important for a 
International Journal of Managing Public Sector Information and Communication Technologies (IJMPICT)

Vol. 6, No. 2, June 2015

good social life, becoming a good wellbeing, looking and feeling better, and a happy healthy life. Patients can get knowledge of new treatments.

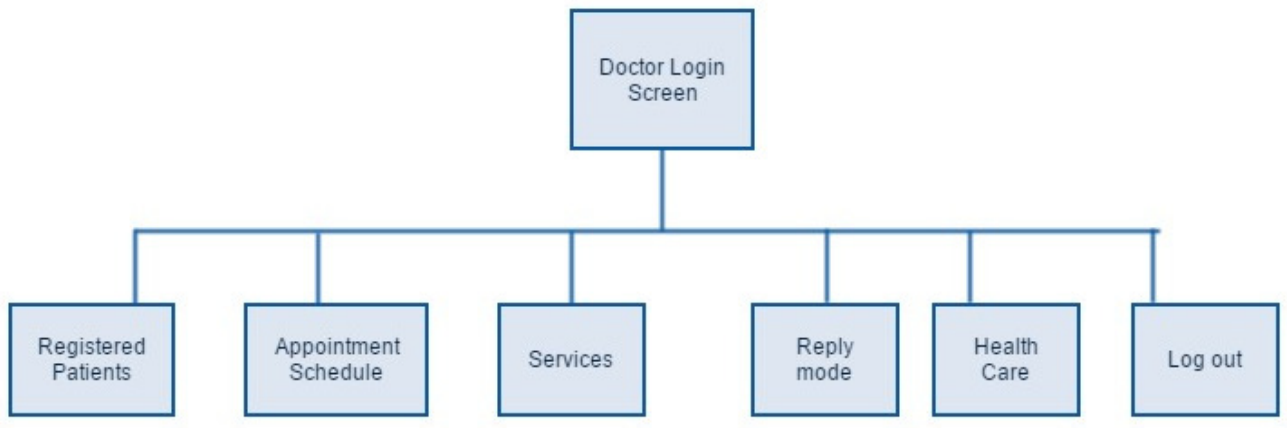

Figure 5. Doctor login module

Figure 5 depicts the basic functionalities of Doctor Login system.

Registered_Patients Module: The Doctor can view all the patients registered to him with all of his details.

Appointment_Schedule Module: The doctor can view the appointment schedule and can set the new appointments accordingly. This module will help in making proper adjustments.

Reply_Mode Module: The reply mode module allows the patient to ask some questions related to the prescribed pills, medicine schedules and other queries. The doctors can reply in yes or no mode.

Table 1. Data Description for Set_Alarm Module

\begin{tabular}{|l|l|l|}
\hline Data Name & Data Type & Description \\
\hline A_Id & Digit & Stores the unique id of the alarm set by the patient. \\
\hline A_Name & String & Stores the description of the alarm. \\
\hline A_Time & Digit & Stores the time of alarm. \\
\hline A_Date & Digit & Stores the date of alarm. \\
\hline A_Medicine_Name & String & Stores the name of the medicine with its description. \\
\hline A_Doctor_Name & String & Stores Doctor's Name. \\
\hline
\end{tabular}

Table 2. Data Description for Get_Notification Module

\begin{tabular}{|l|l|l|}
\hline Data Name & Data Type & Description \\
\hline N_Id & Digit & Stores the unique id of the notification. \\
\hline N_Status & String & Stores the status of the notification. \\
\hline N_Email_Status & String & $\begin{array}{l}\text { Stores the information of the notification sent } \\
\text { through email. }\end{array}$ \\
\hline N_Message_Status & String & $\begin{array}{l}\text { Stores the information of the notification sent } \\
\text { through message. }\end{array}$ \\
\hline N_Message_Content & String & $\begin{array}{l}\text { Stores the information to be shown regarding } \\
\text { notification. }\end{array}$ \\
\hline
\end{tabular}

Tables 1 and 2 show the data description for Set_Alarm and Get_Notification modules. 


\section{COMPARISON WITH OTHER SYSTEMS}

MedsLog, an application only for the iPhone users, is very complex application as compared to others. The users need to spend much more time with the software to understand its functionalities in a proper manner. The main problem with the system is it has "consumed by" box where a user is supposed to fill his username in the provided space. Still the system shows "no people" [12]. In contrast the proposed system is very much user friendly because it is made for the people of all ages. So one can utilize the time in using the system rather than wasting the time in understanding the software. The users can easily manage their profile.

MotionPHR Health Record Manager which is available for $\$ 10$ for full version on Android and iPhone and $\$ 2$ for a Lite version on iPhone, is less rated by the users because of the problem about the flaws in the reminder system and a service that backs up user data [12]. Medsy is also an application which tries to provide medicine remaindering system but it is loaded with less features. If the user is supposed to take a medicine three times a day then this application does not allow to set alarm accordingly. But in our work this disadvantage has been overcome by allowing users to set multiple alarms and notifications [12].

Another application DoseCast is loaded with some good features but it fails to provide notifications if the users do not have a $3 \mathrm{G}$ or Wi-Fi connection [12].Wedjat also serves the same purpose. It can revise the in-take schedule automatically when a dose was missed without the doctor's prescription [8].Because of the implementation of health care module in our system, a user will be provided with a daily health care tip along with the related videos and articles. So the proposed work tries to overcome all the listed disadvantages of other systems.

Table 3. Comparison of the App with three existing Apps

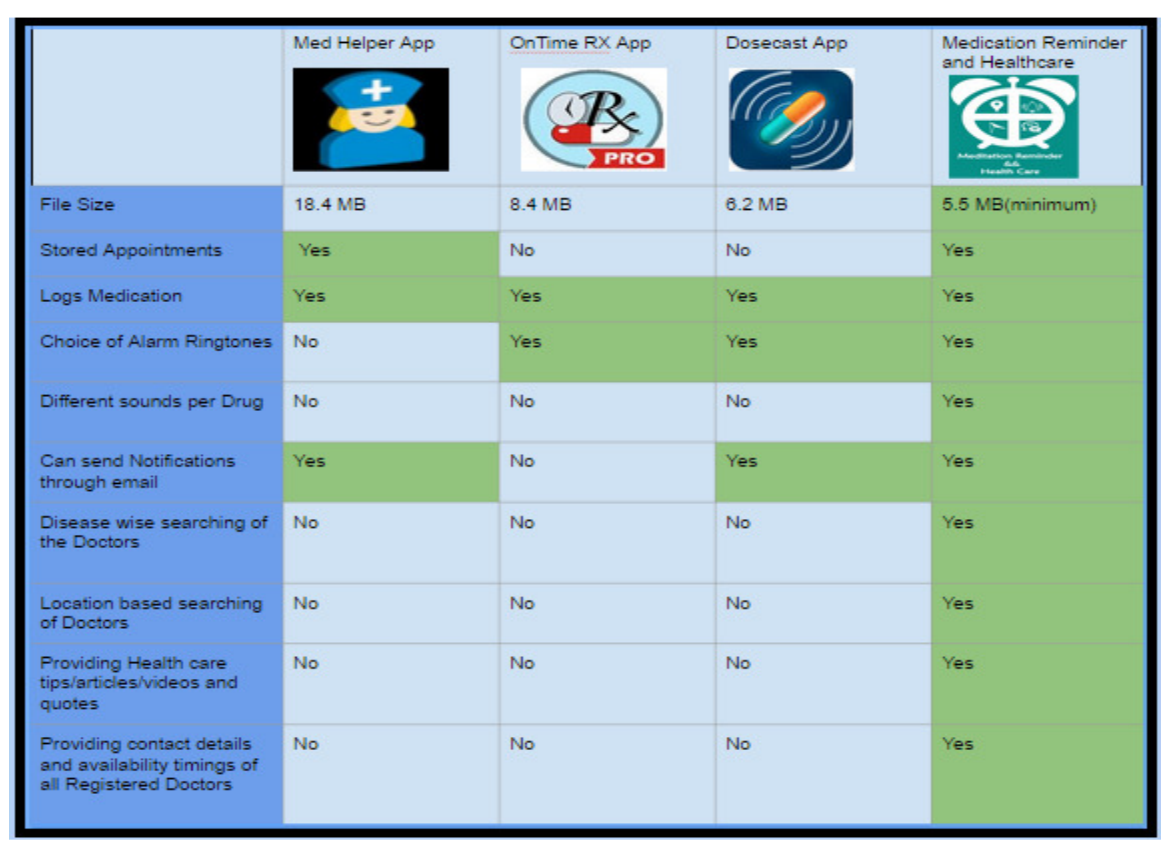


International Journal of Managing Public Sector Information and Communication Technologies (IJMPICT)

Vol. 6, No. 2, June 2015

Table 3 shows a comparison of Medication Reminder and Healthcare App with three other AppsMedHelper App, OnTimeRX App and Dosecast App. Proposed App supports disease wise, area wise (location-based) searching, also provides tips and articles and availability timings of all registered doctors which is not a feature of the remaining three Apps.

\section{ADVANTAGES OF THE PROPOSED SYSTEM}

The users will get the notifications through SMS also. It will provide the information about the medicine timings. The scheduled appointment with the doctor with the contact details including visiting time, venue and availability at different hospitals in case the appointment is missed at the scheduled place. The new appointment will be set accordingly.

The system focuses on improving the rate of attendance at healthcare appointments. The personal phone notifications and reminders are a strong supporting tool in improving medication adherence strategies. The New England Healthcare Institute estimates that $\$ 290$ billion of healthcare expenditures could be avoided if medication adherence were improved [13]. It supports an easy implementation as it is less expensive, reliable, scalable, accessible to anyone with smartphones, and do not require separate devices, packaging or extra hardware. In case if the users phone is switched off and he has set the alarm and the notification is set on, still he will be able to get the notifications through email or message(on his device as well as on other registered number), so it works even when you are running out of the battery. Also a facility of reminding the doctor's next appointment in the system has been focussed.

We have also implemented a navigation system which will allow users to locate the nearest registered hospitals according to their current location. The location based searching of the doctors as well as disease wise searching has been focussed which makes the application more suitable, more user friendly with great features and satisfactory results.

\section{RESUltS}

The application gives reliable reminders, good user interface, nice user experience and it supports many new features supporting medication adherence. We made a survey of 100 people including people of all ages. The following graph (Figure 6) shows the interest of different age groups to the different functionalities provided by the system. This showed that the combination of all the functionalities provided is useful to the people of all ages. Searching doctor disease wise was surveyed beneficial to the people aged around 40 of the total population on whom the survey was made. Again the location based (Area wise) searching of the doctor was a good choice to the population ageing 58. People ageing under 55 found useful the feature of scheduling of the appointment. People of the greater ages are more likely to forget the medicine timings as well as remembering their appointments. The users will get the schedule of medicine in-take time with medicine description, starting and ending date of medicine, notification through message or email, automatic alarm ringing system and navigation system. If the phone is running out of battery then too the system gives the notifications through email and other registered number. So this was found beneficial to the people ageing under 45 as they are more likely to access emails. The automatic alarm ringing feature was proved beneficial to $100 \%$ of the total population. The youths are very much concerned with the new health care awareness and are interested in knowing about new medical techniques being developed every day. So this feature was found 
useful to the youths. Hence the overall system served well in our survey and it truly supports Medication Adherence.

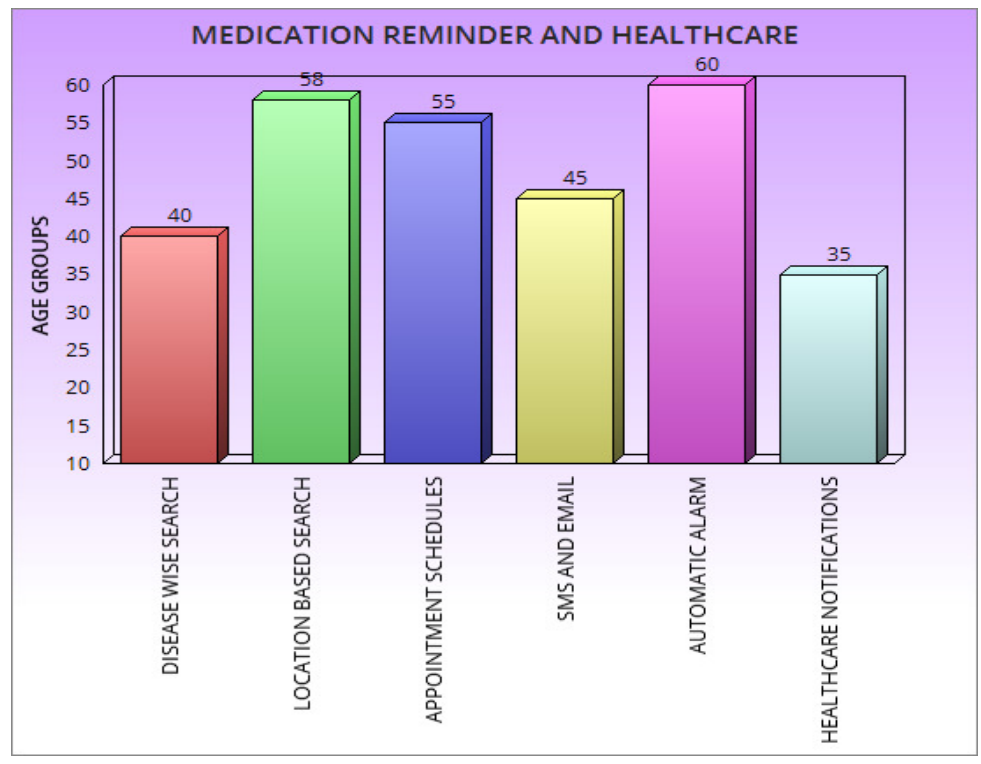

Figure 6. Graph showing different functionalities of the App beneficial to different age groups

\section{CONCluSion AND Future Work}

Many Medication Reminder Systems have been developed on different platforms. Many of these systems require special hardware devices to remind the patients about the medicine in-take timings. Purchasing new hardware devices becomes costly and more time and money consuming. So in the given work an attempt has been made to implement a system which is economical, easily accessible and improves medication adherence. Medication non-adherence reduces the effectiveness of a treatment and imposes a financial burden on health care systems [14] [15]. The patients will get the schedule of medicine in-take time with medicine description, starting and ending date of medicine, notification through message or email, automatic alarm ringing system and navigation system. The scheduled reminder will not suggest any kind of medicine which is not prescribed by the doctor that will assure the safety of the patient and also will avoid wrong dosages. The patients can also search doctors disease wise (depending upon the specialization of the doctor), which provides easy searching facility to the users and saves the time. Doctors can view all the fixed appointments along with date and time, which he fixed and through this he can make new appointment schedules.

We plan to focus on improving the overall performance of the system. Also, interaction between patients and doctors through video calling and secure prescription will be focused upon. Some more ways to achieve medication adherence will be focused. 
International Journal of Managing Public Sector Information and Communication Technologies (IJMPICT) Vol. 6, No. 2, June 2015

\section{REFERENCES}

[1] Park, KeeHyun \& Lim, SeungHyeon, (2012) "Construction of a Medication Reminder Synchronization System based on Data Synchronization”, International Journal of Bio-Science and Bio-Technology, Vol.4, No. 4, pp1-10.

[2] "Smartphone medication adherence apps: Potential benefits to patients and providers", available at: http://www.ncbi.nlm.nih.gov/pmc/articles/PMC3919626/

[3] Slagle, J.M., Gordon, J.S., Harris, C.E., Davison, C.L., Culpepper, D.K., Scott P. and Johnson, K.B., (2011) "MyMediHealth - Designing a next generation system for child-centered medication management", Journal of Biomedical Informatics, Vol. 43,No. 5, pp. 27-31.

[4] Becker, E., Metsis, V., Arora, R., Vinjumur, J.K., Xu, Y. and Makedon, F. (2009) "SmartDrawer: RFID- Based smart medicine drawer for assistive environments", Proc. of Pervasive technologies related to assistive anvironments, June, pp 1-8.

[5] Ammouri, S. and Bilodeau, G.A. (2008) "Face and hands detection and tracking applied to the monitoring of medication intake", Proc. of Canadian Conf. on Computer and Robot Vision, May, pp. 147-154.

[6] Batz, D., Batz, M., Lobo, N.D.V. and Shah, M. (2005) "A computer vision system for monitoring medication intake", Canadian Conf. on Computer and Robot Vision, May, pp. 362-369.

[7] Prasad, B., (2013) "Social media, health care, and social networking", Gastrointest Endosc. Vol. 77, pp 492-495.

[8] Zao, J.K., Wang, M.Y., Peihsuan, T. and Liu, J.W.S., (2010) "Smart Phone Based Medicine In-take Scheduler, Reminder and Monitor", IEEE e-Health Networking Applications and Services (Healthcom), pp $162-168$.

[9] “Android", available at: http://www.openhandsetalliance.com/android_overview.html

[10] Mahmood, R., Mirzaei, N., Malek, S., (2014), "EvoDroid: Segmented Evolutionary Android Apps", FSE'14, November 16-21, 2014, Hong Kong, China

[11] "Medication Adherence", available at: http://circ.ahajournals.org/content/119/23/3028.full

[12] "Healthful Reminders for Medications, Beyond an Apple a Day", available at: http://www.nytimes.com/2010/09/30/technology/personaltech/30smart.html?_r=0

[13] "Thinking Outside the Pillbox: A System-wide Approach to Improving Patient Medication Adherence for Chronic Disease” (2009), A NEHI Research Brief July 2009, New England Healthcare Institute.

[14] Hughes, D. A., Bagust, A., Haycox, A., and Walley, T.O.M. (2001) "The impact of non-compliance on the cost effectiveness of pharmaceuticals: a review of the literature", Health Economics, pp. 601615 .

[15] "Adherence to long term therapies: Evidence for Action" (2003), Report by World Health Organization.

\section{Authors}

Deepti Ameta is currently pursuing B.E. in Computer Engineering from Gandhinagar Institute of Technology, Gujarat Technological University, India. Her areas of interest are Web Development, Database System, Operating System and System Programming.

Kalpana Mudaliar is currently working as an Assistant Professor in Gandhinagar Institute Of Technology, Gujarat Technological University, India. She has an experience of four years. Her research interest include Cellular Netwoking. She is currently working on Location Management and Cellular Networking.

Palak Patel is currently pursuing B.E. in Computer Engineering from Gandhinagar Institute Of Technology, Gujarat Technological University, India. Her research interests include graphics visualisation and learning new programming languages.
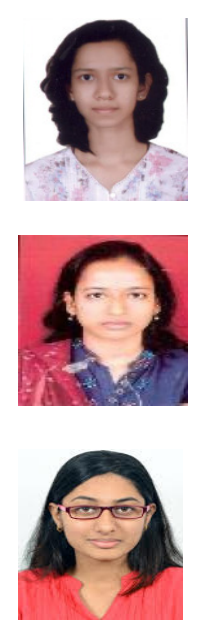